UDK 784.3(436) Mahler

\title{
BEMERKUNGEN ZU GUSTAV MAHLERS LIEDERN
}

\author{
Kurt v. Fis c her (Erlenbach-Zürich)
}

Es gehört mit zu den für Gustav Mahlers Werk konstitutiven Widersprüchen, daß das seinem Wesen nach kleinformatige lyrische Lied sowohl Ausgangs- als auch Zielpunkt der großen symphonischen Schöpfungen des Komponisten darstellt. Dies läßt sich einerseits chronologisch nachweisen, indem sowohl den ersten vier als auch den folgenden Symphonien je bestimmte Liedgruppen zuzuordnen sind. Im "Lied von der Erde» vollzieht sich sodann eine Synthese eigenster Art von Lied und Symphonie. Andererseits aber durchdringen sich die beiden Gattungen auch motivisch und thematisch: Die ersten vier Symphonien enthalten bekanntlich Material aus den "Liedern eines fahrenden Gesellen" (1. Symphonie) und aus den Wunderhornliedern (2., 3. und 4. Symphonie). Weniger offensichtlich, aber ebenso bedeutsam ist der Zusammenhang zwischen den "Kindertotenliedern" und einzelnen andern Rückert-Liedern in den Symphonien 5 bis 9 . Das Verständnis von Mahlers Welt erschließt sich deshalb zu einem we sentlichen Teil aus den Liedern.

Daß sich Mahler spätestens von den frühen Achtzigerjahen bis zur Jahrhundertwende vor allem mit Texten aus "Des Knaben Wunderhorn" auseinandergesetzt hat, ist von ganz entscheidender Bedeutung. Diese Volkslieder, meist jedoch dem Volkslied nachgebildeten und daher durchaus artifiziellen Gedichte aus der 1806-1808 zum erstenmal von Achim von Arnim und Clemens Brentano herausgegebenen Sammlung wurden von Mahler nicht als Kunstprodukte im Sinne von Opera perfecta verstanden. ${ }^{1}$ Für ihn waren dies vielmehr Produkte aus "Natur und Leben"² oder, wie er ein andermal sagt "Felsblöcke, aus denen jeder das Seine formen dürfe«. ${ }^{3}$ So erklärt es sich auch, daß Mahler zuweilen verschiedene Texte aus

${ }^{1}$ F. E. Pamer geht von der nur sehr beschränkt richtigen und insbesondere im Hinblick auf Mahlers Vertonungen irreführenden Voraussetzung aus, dass es sich bei den Wunderhornliedern um Volkslieder handle. Vgl. Pamer F. E., "Gustav Mahlers Lieder", in Studien zur Musikwissenschaft 16 (1929) pg. $116 \mathrm{ff}$. und 17 (1930), pg. $105 \mathrm{ff}$.

${ }^{2}$ Gustav Mahler, Briefe 1879-1911, Berlin, Wien, Leipzig 1924, p. 254.

${ }^{3}$ Mahler-Werfel A., Erinnerungen an Gustav Mahler, Propyläen Verlag 1971; pg. 20 (Einleitung von D. Mitchell). 
der Wunderhornsammlung mischte, Neues dazu erfand und in den Nummern 2 bis 4 der "Lieder eines fahrenden Gesellen" auch eigene Gedichte im Stile der Wunderhornlieder schuf. Durch diese Umdichtungen und, in noch weit höherem Maße, durch ihre Vertonungen sind diese Lieder zum eigensten Bestandteil von Mahlers Personalsphäre geworden; in ihnen spiegelt sich Mahlers ganze Persönlichkeit.

Ein in dieser Hinsicht überaus aufschlußreiches Beispiel ist das erste der Gesellenlieder: "Wenn mein Schatz Hochzeit macht». Aus den acht Zeilen der Wunderhorn-Vorlage wird bei Mahler ein mehr als doppelt so langes Gebilde, das, für den Komponisten außerordentlich bezeichnend, die gebundene Form stellenweise prosaartig erweitert. So heißen die ersten vier Zeilen bei Arnim-Brentano:

"Wann mein Schatz Hochzeit macht, hab' ich einen traurigen Tag, geh' ich in mein Kämmerlein, wein' um meinen Schatz.k

Und bei Mahler:

"Wenn mein Schatz Hochzeit macht, fröhliche Hochzeit macht, hab' ich meinen traurigen Tag!

Geh' ich in mein Kämmerlein, dunkles Kämmerlein!

Weine! Wein'! um meinen Schatz, um meinen lieben Schatz!»

Wesentlich an Mahlers Textbearbeitung dieser ersten Strophe sind die Intensivierung des Ausdrucks (man vergleiche die vielen Ausrufzeichen) und der Ausbruch aus lyrischer Geschlossenheit in Richtung einer nach innen wie nach außen offenen Form. Durch Zufügung von Adjektiven werden Konstraste verstärkt: "fröhliche Hochzeit», "dunkles Kämmerlein". Durch die Umwandlung von "Hab' ich einen traurigen Tag" $\mathrm{zu}$ »Hab' ich meinen traurigen Tag", aber auch durch Wiederholung des Verbums "weinenı kommt der für Mahler so wesentliche Welt-Ich-Konflikt zum Ausdruck. Noch deutlicher wird dies in Mahlers mehrzeiligem Einschub in die zweite Wunderhornstrophe, wo sich die schöne Welt als trügerischer Traum erweist. Zugleich mit dieser Einschiebung wird die zweistrophige Vorlage zum dreiteiligen Lied umgeformt: Auf die erste vierzeilige Strophe wann mein Schatz Hochzeit macht" folgt in der Wunderhornsammlung die folgende zweite, ebenfalls vierzeilige Strophe:

"Blümlein blau, verdorre nicht, du stehst auf grüner Heide.

Des abends, wenn ich schlafen geh', so denk ich an mein Lieben."

Im Anschluß an die erste Zeile dieser Strophe, die wiederum durch Textwiederholungen ins Emphatische gesteigert ist ("Blümlein blau! Blümlein blau! Verdorre nicht! Verdorre nicht! «) bringt Mahler zunächst eine weitere Vertiefung und Variierung des in der ersten Zeile geäußerten Ge- 
dankens, indem er, in Analogie zur ersten Zeile hinzufügt: "Vöglein süß! Vöglein süß!«. Mit der dritten Zeile übernimmt er, dem Einschub entsprechend leicht abgeändert, die zweite Zeile der Vorlage: "Du singst auf grüner Heide». Anschließend dichtet Mahler wiederum eine Zeile dazu: nAch! wie ist die Welt so schön! Ziküth!». Diese vier Zeilen Mahlers sind als kontrastierender, eine heile Welt der Natur vortäuschender Mittelteil in der Tonart Es-dur den beiden in d-moll stehenden Eckteilen gegenibergestellt. Das eigenartige Wort "Ziküth" scheint Mahlers eigene Erfindung zu sein, ein Wort, das offenbar als "Naturlaut" zu verstehen ist, ein Begriff, der sich oft auch in den Symphonien findet. Auf den Mittelteil folgt eine dritte Strophe, deren zwei letzte Zeilen wiederum den Text aus der zweiten Wunderhornstrophe übernehmen: "Des abends, wenn ich schlafen geh' / denk ich an mein Leide», wobei das versöhnliche "Lieben" der Vorlage - das ganze Gedicht steht in der Wunderhornsammlung unter den "Tanzreimen"! - von Mahler in "Leide" verwandelt wird. Diesen zwei letzten Zeilen sind nun aber vom Komponisten drei neu gedichtete Zeilen vorangestellt, welche recht eigentlich den Kerngedanken des ganzen Liedes enthalten:

"Singet nicht! blühet nicht!

Lenz ist ja vorbei!

Alles Singen ist nun aus!»

Musikalisch nimmt die dritte Strophe wieder das Material der ersten auf, ohne allerdings genau zu wiederholen. Zusammenfassend läßt sich sagen, daß die Gegensätze heile und beschädigte Welt, Schönheit und Leid, Singen und Nicht-mehr-Singer gegenüber der Vorlage wesentlich betont und zum Teil sogar neu eingeführt sind und damit den Gehalt des Wunderhornliedes ganz entscheidend verändern.

Von diesem Lied aus ist nun nach den geschichtlichen Rückbezilgen zu fragen. Hier bietet sich ein anderer Wiener-Komponist an: Franz Schubert, ${ }^{4}$ der besonders in seinen beiden großen Liedzyklen "Die schöne Müllerin" und "Winterreise" den Konflikt von schönem Traum und harter Realität mit den Worten seines Textdichters Christian Müller gestaltet hat; so z. B. im "Frühlingstraum" aus der "Winterreise»:

"Ich träumte von bunten Blumen,

so wie sie wohl blühen im Mai,

...

Und als die Hähne krähten,

da ward mein Auge wach,

da war es kalt und finster,

es schrien die Raben vom Dach."

4 Zur Beziehung Mahler-Schubert vgl. Gerlach R., "Mahler, Rückert und das Ende des Liedes", in Jahrbuch des Staatl. Instituts für Musikforschung Preussischer Kulturbesitz, 1975, p. 7 ff. und insbesondere p. 18 fl. - Vgl. ferner Pamer, a. a. O. II, 121, wo allerdings die Beziohungen der beiden Komponisten bloss in Äusserlichkeiten gesehen werden. 
Von diesem Lied aus läßt sich der Faden wiederum zurück zu Mahler spinnen, nicht nur zum ersten Gesellenlied, sondern auch zum vierten: "Die zwei blauen Augen«. Wie nahe beisammen stehen inhaltlich, aber auch musikalisch doch Schubert-Müllers Strophe:

wDoch an den Fensterscheiben, wer malte die Blätter da?॥

und die letzte Strophe des von Mahler selbst gedichteten vierten Liedes des Gesellenzyklus:

"Auf der Straße steht ein Lindenbaum,

da hab' ich zum ersten Mal im Schlaf geruht!»

Und nun kommen wir nochmals auf das erste Lied dieses Zyklus zurück, in dessen Zentrum die Zeile vom Ende allen Singens steht. Auch Schubert wußte ja um dieses "Alles Singen ist nun aus": Der Leiermann am Schluß der "Winterreise" dreht seine Leier zwar noch, doch

"Keiner mag ihn hören, keiner sieht ihn an»

und das Lied endet mit der resignierten Frage

"Willst zu meinen Liedern deine Leier drehn?"

Unmittelbar an dieses letzte Lied von Schuberts Zyklus knüpft Mahler auch musikalisch in seinem ersten Gesellenlied an. Gemeinsam sind beiden Stücken der Orgelpunkt (bei Mahler ab Takt 5 dieselbe leere Quinte mit Vorschlagsnote wie bei Schubert), die Sechzehntelfigur auf Taktbeginn, der synkopierende Akzent (bei Schubert im vierten und fünften Takt, bei Mahler in den Takten 1 bis 4 der Orchesterfassung) und eine jede Entwicklung verhindernde Reihung von Taktgruppen.
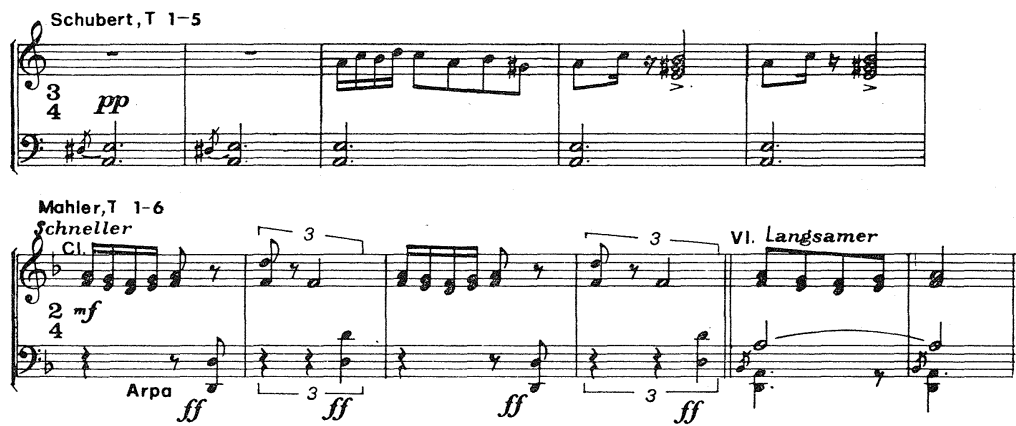

Die bei Schubert gewissermaßen eingefrorene Musik zerfällt bei Mahler in ständigen Tempo- und Metrumwechsel. Daß eine bewußte oder unbewußte Affinität zwischen den beiden Stücken besteht, mag schließlich auch eine Briefstelle des neunzehnjährigen Mahler andeuten. Im Zusammenhang mit der nie vollendeten Oper "Herzog Ernst von Schwaben» schreibt Mahler im Jahre 1879 an seinen Freund und Textdichter der geplanten Oper, Josef Steiner: "Da ziehen die blaßen Gestalten meines Lebens wie 
der Schatten längst vergangenen Glücks an mir vorüber, und in meinen Ohren erklingt das Lied der Sehnsucht wieder ... und dort steht der Leiermann und hält in seiner dürren Hand den Hut hin und in den verstimmten Tönen hör' ich den Gruß Ernst's von Schwaben.. ${ }^{5}$

Während die Gesellenlieder höchstwahrschenlich zwischen 1883 und 1885 als Klavierlieder entstanden sind und erst nachträglich für Orchester gesetzt, bezw. vollendet worden sind, waren die jüngeren Wunderhornlieder aus den Jahren 1892-1901 (im Gegensatz zu den älteren der Jahre 1887-1890) von Anfang an als Orchesterlieder konzipiert. ${ }^{6}$ Diese Feststellung ist wichtig: Sie verbietet es endgültig, diese Stücke, wie im Grunde auch schon die Gesellenlieder, dem privaten, bürgerlich-häuslichen Kreis und noch viel weniger dem schlichten Volkslied zuzuorden. Diese Lieder repräsentieren eine ganz eigene Welt, die nur wenig mit dem übrigen Liedschaffen des ausgehenden 19. Jahrhunderts zu tun hat. Ihre geistigen Wurzeln reichen, wie gleich nochmals zu zeigen sein wird, viel eher auf Schubert zurück. Musikalisch-formal ist hier allerdings noch ein anderer Komponist zu nennen, dessen kritisches Außenseitertum auch musiksoziologisch gewisse indirekte Beziehung zu Mahler erkennen lässt: Hector Berlioz und dessen $\mathrm{zu}$ seiner Zeit völlig isoliert dastehende, allerdings aus Klavierliedern hervorgegangene Orchesterlieder. Es spricht für das feine musikalische Gefühl des Wienerkritikers Eduard Hanslick, wenn er diese lose musikalische Verwandtschaft zwischen Mahler und Berlioz schon anläßlich eines Mahlerlied-Aufführung im Jahre 1900 herausgespürt hat. Hanslick schreibt von diesen Liedern, es handelt sich um zwei Gesellen- und drei Wunderhornlieder, sie seien schwer zu klassifizieren, weder Lied, noch Arie, noch dramatische Szene, und doch besäßen sie etwas von allen diesen Typen; ihre Form erinnere, mehr als an irgend etwas anderes, an Berlioz's Orchesterlieder. ${ }^{7}$

Zentrales, wenn auch keineswegs ausschliessliches Thema der von Mahler vertoten Wunderhornlieder ist der Soldat. Der fahrende Gesell ist zur entpersönlichten, den fremden Mächten des Marsches, der Trommel und des Todes gehorchenden Gestalt geworden. Damit wird etwas von dem gestalten, das unmittelbar mit der ganzen Atmosphäre der vom Untergang gezeichneten Donaumonarchischen Vorkriegswelt zusammenhängt. Adorno hat es so formuliert: "Mahlers Musik ist Traum des Individuums vom unaufhaltsamen Kollektiv. Zugleich aber drückt sie objektiv aus, daß Identifikation mit ihm unmöglich ist. ${ }^{8}$ Von hier aus ergibt sich eine weitere Affinität zu Schubert, in dessen Musik, schon allein vom Klangbild her, marschähnliche Rhythmen vielfach eine ganz zentrale Rolle spielen. Was bei Schubert teils Resignation, teils dunkle Verfallenheit und Hinweis auf brüchige Stellen dieser Welt bedeutet, wird in Mahlers Wunderhornliedern

\footnotetext{
${ }^{5}$ Bricfe, p. $7 / 8$.

${ }^{6}$ Zur Datierung vgl. Mitchell D., Gustav Mahler-The Wunderhorn Years, London 1975, p. 91 ff. u. 127 ff.; vgl. ferner Tibbe M., Lieder und Liedelemente in instrumentalen Symphoniesätzen Gustav Mahlers, München 1971. p. 125 ff.

7 Vgl. Mitchell, a. a. O., p. 431.

' Adorno Th. W., Mahler, Frankfurt (Suhrkamp) 1969, p. 50.
} 
(aber auch in den Symphonien) als kritisch und vielfach ironisch ausgetragener Konflikt gestaltet. Symbolhaft hiefür steht, neben der oft höchst eigenartigen Metrik Mahlers, der ständige, auf viel kleinerem Raum und wesentlich häufiger als bei Schubert sich abspielende Dur-moll-Wechsel. Und gerade hier setzt bei Mahler nun auch das Neue, über Schubert hinausgehende und weit in die Zukunft weisende an: Alles ist dem Prinzip der Veränderung unterworfen, wweil", wie Mahler zu Natalie Bauer-Lechner gesagt hat, win der Musik das Gesetz des ewigen Werdens, ewiger Entwicklung liegt - wie die Welt, selbst am gleichen Ort, eine immer andere, ewig wechselnde und neue ist .9 Mahler glaubt an die Möglichkeit von Veränderungen. Nie weiß man deshalb in seiner Musik wie es weitergehen wird. Jeder Takt kann Unvorhergesehenes bringen. Aus diesem Grunde ist auch seine ihm eigenste Liedform das frei, oft sehr frei variierte Strophenlied. Ton-für-Ton-Wiederholungen ganzer Abschnitte werden von Mahler als starr, als Symbole von Unveränderbarkeit abgelehnt. Deshalb wohl auch hat er in seiner Dirigierpartitur von Schuberts grosser C-dur Sym. phonie im Andante ganze von Schubert ausgeschriebene Wiederholungspartien herausgestrichen. ${ }^{10}$ Für ihn ist, wie er einmal sagt, "jede Wiederholung ... Lüge». ${ }^{11}$

Doch nun zurück zu den Wunderhornliedern. Als Beispiel diene das von Mahler aus den zwei Gedichten "Bildchen" und "Unbeschreibliche Freude« zusammengesetzte Lied ${ }^{12}$ mit dem vom Komponisten der letzten Strophe entnommenen Titel "Wo die schön Trompeten blasen". Auch hier gibt es wieder Anklänge an Schubert, Anklänge sowohl melodischer wie auch rhythmischer, aber auch inhaltlicher Art. Diesmal aber stammt das Bezugsstück nicht aus der "Winterreise», sondern aus der "Schönen Müllerinu. Es handelt sich um das Lied "Trockene Blumen". Die folgenden Beispiele sollen diese Beziehungen deutlich werden lassen:

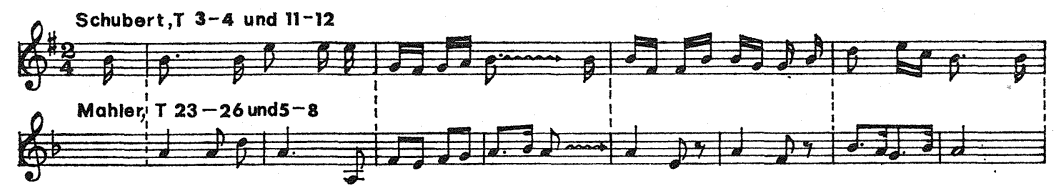

Auch textlich zeigen die beiden Lieder Gemeinsamkeiten: Dem Grabeshügel bei Schubert (")und wenn sie wandert am Hügel vorbei«) entspricht der "gruine Rasen" in der letzten Strophe des Mahler-Liedes. Nun aber endet dieses Lied Schuberts, im Gegensatz zum "Leiermann", nicht in tödlicher Resignation, sondern erhebt sich in der letzten Strophe zu einer Vision, ausgedrückt durch den Tongeschlechtswechsel von e-moll nach E-dur.: "Der Mai ist kommen, der Winter ist aus." Damit läßt es, um mit

- Bauer-Lechner N., Erinnerungen an Gustav Mahler, Leipzig, Wien, Zuirich 1923, p. 119.

${ }_{10}$ Vgl. Andraschke P., "Die Retuschen Gustav Mahlers an der 7. Symphonie von Franz Schubert«, Archiv f. Musikwiss. XXXII (1975), pg, $111 \mathrm{ff}$.

it Bauer-Lechner, a. a. O.pg. 138.

12 Vgl. Pamer F. E., a.a. O., I, 124 ff. 
Mahlers Worten zu reden, etwas vom "Gesetz des ewigen Werdens« hörbar werden. Auch Mahlers Lied von den "schönen Trompeten" - das uschön" ist hier durchaus ironisch $\mathrm{zu}$ verstehen - stellt eine dunkle und beschädigte Welt einer hellen gegenüber, indem marschartige, gewissermaßen männliche Abschnitte lyrisch-weiblichen gegenübergestellt sind. Die Form ist eine Bogenform ABACABA, wobei die A-Teile dunkle Fanfarenklänge im 2/4-Takt, die B-Teile eine liedhafte Melodie im 3/4-Takt zeigen. Daß die A-Teile bei ihrer Wiederholung nicht identisch sind, ist für Mahler wohl selbstverständlich. Umso auffallender ist deshalb die, wenn zwar auch nicht vollständige, so doch weitgehende Identität der beiden B-Teile, die gerade mit solcher Wiederholung heile Welt vortäuschen. Doch Mahler arbeitet in diesem Lied auch mit Widersprüchen: Es sprechen, nach Art eines Rollengedichtes, ein Knabe und ein Mädchen. Das Auffallende besteht nun aber darin, daß die marschhaften männlichen Teile (mit Ausnahme des Schlußteils) den Worten des Mädchens, die lyrisch-weiblichen B-Teile dagegen den Worten des Knaben zugeordnet sind. Erst im letzten A-Teil werden Krieg und Tod, d.h. die volle Realität, mit den Worten des Knaben verbunden: 13

"Ich zieh' in Krieg auf grüne Heid';

die grüne Heide, die ist so weit!

Allwo dort die schönen Trompeten blasen,

da ist mein Haus von grünem Rasen!«

Aber auch diese Strophe ist doppelbödig: Die kriegerische Fanfarenmelodie der Singstimme klingt eigenartig verfremdet. Die Realität des Schlus-

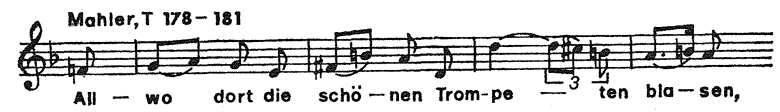

ses wird damit ihrerseits wiederum kritisch in Frage gestellt und durch den dieser Strophe entnommenen Liedtitel "Wo die schönen Trompeten blasen« aufs ganze Lied übertragen.

Noch einmal, mit den letzten zwei, ungefähr gleichzeitig mit fünf Rükkert-Vertonungen entstandenen Wunderhornliedern der Jahre 1899 und 1901, "Rewelge" und "Der Tamboursg'sell" bricht das Motiv des Todesmarsches mit ganzer Gewalt im Liede durch. wMarschierend, in einem fort" steht über "Rewelge»; Satz und Instrumentation sind für Mahler merkwürdig massiv und undurchlässig. Ironisch wirkt der eigenartige, im Gegensatz zum üblichen Dur-moll stehende moll-Dur-Schluß (eine Art von doppelter Verfremdung).

Als nächste Liedgruppe folgen in den Jalren 1900 bis 1904 die "Kindertotenlieder" nach Texten von Friedrich Rückert. Wiederum ist der Tod thematisiert. Doch hat sich die musikalische Sprache und damit auch die semantische Bedeutung der Musik gewandelt. An die Stelle der schweren

${ }^{13}$ Die unglückselige Mode, dieses Lied im Wechselgesang einer Männer- und einer Frauenstimme vorzutragen zerstört die von Mahler intendierte Dialektik. 
und geballten Klänge von "Rewelge" tritt nun ein äußerst durchsichtiger und kammermusikalischer Satz von polyphoner Faktur. Mahlers Polyphonie hat freilich nichts mit retrospektivbarocker Polyphonie $\mathrm{zu}$ tun $_{\mathrm{d}}$ In ihrem wesentlich von Klangschichten und Klangfarben bestimmten Satz stellt sie vielmehr eine neue Stufe der Reflexion im Denken des Komponisten dar. Die Singstimme ist in gewissem Sinne entsubjektiviert; sie verhält sich wie ein Instrument, so daß auch jederzeit Stimme und Instrument austauschbar sind: instrumentale und vokale Motivik gehorchen denselben Prinzipien. Eine weitere Eigenart dieser Lieder ist die wichtige Rolle, welche Celesta und Harfe spielen. Mit den in der sechsten Symphonie eingeführten Herdenglocken zusammen repräsentieren diese Instrumente Weltferne und damit nein Sehnen über die Dinge dieser Welt hinaus «. ${ }^{14}$ Diesem Gedankenkreis sind auch die fünf von Mahler vertonten Rückerttexte zugeordnet; so etwa im ersten Lied:

nDu mußt nicht die Nacht in dir verschränken, mußt sie ins ewge Licht versenken.॥

oder Im vierten Lied:

nO, sei nicht bang, der Tag ist schön!

Sie machen nur den Gang nach jenẹn Höh'n."

So wie die Symphonien eins bis vier von der Wunderhornthematik bestimmt sind, so stehen, in freilich musikalischformal ganz anderer Weise, die lolgenden Symphonien mit den "KKindertotenliedern" in Verbindung. Daß aber die Kindertotenlied-Motivik und Klangfarbe, der Kindertotenliederton, auch schon vor der Komposition des Liederzyklus auftreten, zeigen die Takte (und insbesondere die Sexten) vor Ziffer 9 des Nietzsche-Satzes der dritten Symphonie. Tonsprachliche Elemente der Kindertotenlieder sind also offenbar schon um 1895/96 vorhanden. Es bestätigt sich hier, was Adorno als die große Wesenseinheit des gesamten Mahlerschen Oeuvres versteht: "Alle Werke Mahlers kommunizieren unterirdisch miteinander wie die Kafkas durch Gänge des von diesem geschilderten Baus. ${ }^{15}$ Auf die enge Beziehung zwischen dem Adagietto der 5. Symphonie und dem Rückert-Lied "Ich bin der Welt abhanden gekommen" ist in der Literatur schon mehrfach hingewiesen worden.16

Die Art des Zusammenhangs zwischen Lied und Orchesterwerk hat sich gegenüber den vier ersten Symphonien entscheidend gewandelt. Wenn in diesen ganze Lieder oder Liedteile die Grundlage von Symphoniesätzen bildeten, so sind es jetzt nur noch einzelne Liedfragmente und Anklänge, die, meist vermutlich nicht einmal als Zitate zu verstehen, auftauchen und wieder verschwinden. An die Stelle der tbernahme von Liedern tritt jetzt eine im Untergrund wirkende Materialgemeinschaft von Lied und Symphonie. Vollends zur Einheit verschmolzen sind die beiden Gattungen im

${ }^{14}$ Bauer-Lechner N., a.a.O.,pg. 119: "Die Musik muss immer ein Sehnen enthalten, ein Sehnen über die Dinge dieser Welt hinaus».

us Adorno, a. a. O.,pg. 77.

16 Vgl. u. a. Gerlach, a. a. O., pg. 20. 
"Lied von der Erde». Daß diese Deutung richtig ist, ergibt sich nicht nur aus dem Titel und aus der ja schon in den früheren Symphonien und wierderum in der Achten anzutreffenden Verbindung vokaler und instrumentaler Elemente, sondern vor allem im Abbau des Monumentalen. Einzelne kammermusikalische Partien lassen die gedankliche Verbindung mit den Kindertotenliedern erkennen. Solches wird besonders im letzten Satz, "Abschied", deutlich, dort etwa, wo von den "ewig blauen lichten Fernen" die Rede ist. Das unendliche Male wiederholte »ewig, ewig«, zusammen mit den auch im Kindertotenzyklus mehrfach erscheinenden, regelmäßig pendelnen Klängen von Celesta und Harfe, läßt diese Beziehung auch klanglich evident werden. Hier schließt der erste Satz der Neunten unmittelbar an: Motivik und Instrumentation sowie der fragmentarische Charakter der musikalischen Struktur lassen den Anfang der Symphonie aus den letzten Takten des "Liedes von der Erde" herauswachsen.

Im letzten Satz der Neunten endlich, den mit "Finale» zu bezeichnen Sakrileg wäre, erscheint dreimal ein Zitat aus dem vierten Kindertotenlied. Wie Monika Tibbe überzeugend gezeigt hat, kann kaum ein Zweifel daruber bestehen, daß an diesen Stellen von Mahler nicht nur melodisches Zitat oder Materialgemeinschaft, sondern zugleich auch, zwar unausgesprochen wortlos, textliches Zitat gemeint sein muß.17 Dies wird besonders dort deutlich, wo, zweiundzwanzig Takte vor dem im dreifachen Pianissimo verklingenden und verstummenden Schluß, zwanzig Melodietöne aus der dritten Strophe des vierten Kindertotenliedes in der ersten Violine "mit inniger Empfindung" erklingen. Der Text der zitierten Melodie heißt im Kindertotenlied: "...im Sonnenschein / Der Tag ist schön auf jenen Höh'n«:

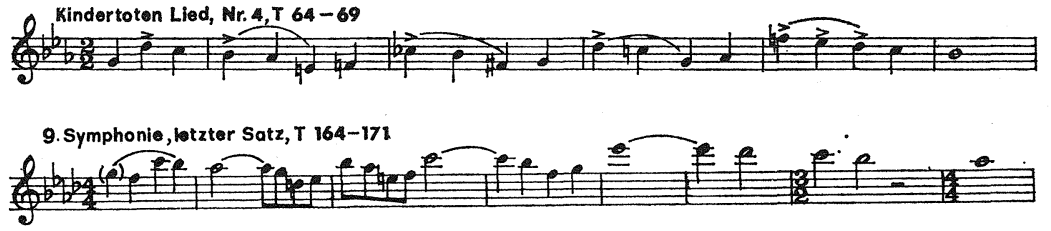

Diese utopische Schau in die ferne Zukunft liegt als Inhalt auch dem Ende der 9. Symphonie zugrunde. Anders ist dieses im späten Schaffen Mahlers ganz außergewöhnliche Zitat - außergewöhnlich auch an Deutlichkeit und Ausdehnung - nicht zu interpretieren.

Sind Mahlers Lieder und Symphonien, wie Adorno in den letzten Sätzen seines Mahler-Buches schreibt, wirklich "ohne Verheißung", als "Balladen des Unterliegens" zu verstehen, ${ }^{18}$ und ist wirklich sinnliche Schönheit bei Mahler nur noch als Erinnerung möglich? Werke wie "Rewelge», "Der Tamboursg'sell" und vor allem die 6. Symphonie scheinen diese These zu bestätigen. Ihr widersprechen aber andere Werke und insbesondere solche aus der Spätzeit: Kindertotenlieder, "Das Lied von der Erde» und im be-

$17 \mathrm{Vgl}$. Tibbe in der in Anm. 6 genannten Arbeit, pg. $120 \mathrm{ff}$.

18 Adorno, a. a. O., pg. 216. 
sonderen der letzte Satz der 9. Symphonie. In diesen Werken wird mit oft einfachsten Mitteln des schönen Klanges Unsagbares ausgesagt. Gewiß: Erinnerung und Abschied auch da, aber zugleich ist hier "das Sehnen uiber die Dinge dieser Welt hinaus« spürbar. Verstehen wir es recht: Es geht hier nicht um eine affirmativ metaphysische Deutung von Mahlers Musik, denn nie versucht Mahler - vielleicht mit gewissen Ausnahmen in der Achten der Transzendenz habhaft $\mathrm{zu}$ werden. Und doch ist seine Musik keineswegs, einem verlorenen Paradiese nachtrauernd, allein nach rückwärts gewandt. Vielmehr postuliert sie immer wieder, aber stets mit äußerster Behutsamkeit, eine offene Zukunft, offen im Sinne einer veränderbaren Welt, in der Schönheit vielleicht einmal wieder möglich sein könnte. An der Wahl der Liedtexte und an den kompositorischen Mittel läßt sich der Weg Mahlers aus den Erinnerungsbildern des Gesellenzyklus über die Aussagen von einer beschädigten Welt in einige Wunderhornliedern bis hin zur utopischen Öffnung der späten Lieder und Symphonien verfolgen. Es ist gewiss kein Zufall, wenn Mahler im Winter 1909 an Bruno Walter den Satz geschrieben hat: "Ich sehe alles in einem so neuen Licht.»19

\section{POVZETEK}

Liricne pesmi, ki so $\mathrm{v}$ bistvu miniaturne oblike, predstavljajo izhodišce in cilj velikih Mahlerjevih simfoničnih stvaritev. Obe zvrsti se $v$ njegovem opusu motivično in tematsko prepajata. Vsekakor je odločilnega pomena, da se je Mahler že vse od osemdesetih let prejšnjega pa do začetka sedanjega stoletja ukvarjal besedili zbirke "Des Knaben Wunderhorn" ("Dečkov čudežni rog»). Te pesmi, ki so napisane po vzoru ljudskih, so bile zanj "kamniti bloki, iz katerih si lahko vsakdo izoblikuje svoje». Zato je razumljivo, da je Mahler včasih mešal iz njih različne tekste ali pa da jim je mestoma dodal tudi kaj lastnega. V ciklu "Lieder eines fahrenden Gesellen॥ ("Pesmi popotnega tovarišaı) pa je celo sam spesnil 2., 3. in 4. pesem $v$ stilu omenjene zbirke. $S$ takšnimi prepesnitvami in še bolj z uglasbitvijo so postale te pesmi najbolj lasten del Mahlerjeve osebnosti. V tej zvezi sta značilna prvi in cetrti spev iz cikla "Lieder eines fahrenden Gesellenu, ki kažeta določeno vsebinsko in glasbeno sorodnost $\mathbf{s}$ Schubertovima pesmima "Der Frühlingstraum" ("Pomladni sen") in "Der Leiermann" ("Lajnar"). Medtem ko so "Pesmi popotnega tovariša" nastale v letih 1883-1885 kot samospevi s klavirjem in so bile šele pozneje instrumentirane, je bil mlajši ciklus «Des Knaben Wunderhorn॥ (1892-1901) že od vsega začetka zasnovan z orkestralno spremljavo. Te pesmi predstavljajo povsem svojstven svet in skoro nimajo zveze $\mathbf{s}$ pesemsko ustvarjalnostjo poznega 19. stoletja. Njihove duhovne korenine izvirajo iz Schuberta, $v$ glasbeno oblikovnem pogledu pa je seveda treba opozoriti na orkestralne pesmi H. Berlioza. Osrednja, čeprav ne edina tema tega cikla je vojak, $\mathrm{ki}$ je poslušen čudnim silam pohoda, bobna in smrti. Od tod izhaja določena sorodnost $\mathrm{s}$ Schubertom, $\mathrm{v}$ čigar glasbi imajo ritmi koračnice važno vlogo. Tako kaže pesem "Wo die schönen Trompeten blasen" (Kjer igrajo lepe trobente) reminiscence $\mathrm{v}$ ritmičnem, melodičnem in vsebinskem pogledu S Schubertovo "Trokkene Blumen« ("Suhe cvetke»). Z razliko od "Lajnarja» pa se ta pesem ne konča $\mathrm{z}$ resignacijo, ampak se $\mathrm{v}$ zadnji kitici povzpne do svetle vizije. Podobno kot tu si stojita $\mathrm{V}$ Mahlerjevi "Wo die schönen Trompeten blasen " nasproti mračen in svetal svet ter kontrastirajo odseki $v$ krepkem ritmu koračnice $\mathrm{z}$ nežno lirskimi. Smrt je spet tema naslednjega cikla "Kindertotenlieder» (»Pesmi za mrtvo decou, 1900-1904), vendar se je spremenila glasbena govorica in njen semantični pomen. Kompozicijski stavek je polifon in komorno prosojen. Pevski glas je v določenem smislu desubjektiviran in se ponaša kot instrument, tako da je mogoce $v$ vsakem

${ }^{19}$ Briefe, pg. 414. 
trenutku zamenjati glas in instrument. Važno vlogo dobita celesta in harfa. Te pesmi so odločujoče za poznejšo Mahlerjevo simfonično ustvarjalnost, tako kot so bile pesmi iz cikla "Des Knaben Wunderhorn" pomembne za prve štiri simfonije. Seveda pa je tokrat zveza med enim in drugim področjem dokaj drugačna. Če so bile prej cele pesmi ali njihovi deli osnova simfoničnih stavkov, se zdaj pojavljajo $\mathrm{v}$ njih le posamezni fragmenti pesmi ali le reminiscence, $\mathrm{ki} j \mathrm{jh}$ niti ne moremo poịmovati kot citate. Končno in najtesnejšo sintezo pesmi in simfonije je Mahler dosegel v ciklu "Das Lied von der Erde» ("Pesem o zemljii). Pravilnost takšnega tolmačenja potrjuje predvsem eleminiranje monumentalnega. Tu očitujejo posamezni komorni pasusi jasno miselno zvezo s deco», iz zadnjih taktov poznejšega cikla pa tudi razlocno izhaja začetek MahIerjeve 9. simfonije. 